\title{
ANÁLISIS COMPARATIVO DEL REGISTRO LÍTICO DEL CONJUNTO DE PARAPETOS GUITARRA 10 (MESETA DEL LAGO GUITARRA, SANTA CRUZ, ARGENTINA)
}

\section{COMPARATIVE ANALYSIS OF HUNTING BLINDS` LITHIC RECORD FROM SITE GUI 10 (GUITARRA LAKE PLATEAU, SANTA CRUZ, ARGENTINA).}

\author{
Gisela Cassiodoro ${ }^{1}$ y Josefina Flores Coni ${ }^{2}$ \\ 1 CONICET-UBA-INAPL. 3 de febrero 1378, CABA, Argentina gcassio@hotmail.com \\ 2 CONICET-INAPL. 3 de febrero 1378, CABA, Argentina. josefinafloresconi@gmail.com
}

Presentado: $27 / 08 / 2018$

Aceptado: 26/04/2019

\section{Resumen}

En las mesetas patagónicas existe una alta frecuencia de estructuras de piedra denominadas parapetos. El análisis de dichas estructuras y de los conjuntos tecnológicos asociados resulta relevante para abordar las características de la ocupación de estos espacios. Así, se busca evaluar la variabilidad de un conjunto de 11 parapetos del sitio Guitarra 10 (meseta del lago Guitarra, provincia de Santa Cruz, Argentina). El análisis comparativo del registro lítico es realizado desde dos aspectos. Por un lado, se focaliza en establecer las posibles diferencias en el uso del espacio interno y externo de las estructuras y por otro, se centra en dar cuenta de la variabilidad relacionada con la localización altitudinal de los parapetos dentro del sitio. Es esta última la que presenta un mayor aporte, permitiendo ampliar la discusión sobre las características de la utilización de estas estructuras.

Palabras clave: Parapetos, tecnología, uso del espacio, Patagonia.

\begin{abstract}
There is a high frequency of stone structures called parapetos in plateaus from Patagonia. The analysis of these structures and the artefactual assemblages related to them are relevant to study the characteristics of the occupation of this kind of environments. Thus, we seek to evaluate the variability of a group of 11 hunting blinds from site Guitarra 10 (Lake Guitarra plateau, Santa Cruz province, Argentina). A comparative analysis of lithic technology is carried out based on two aspects. Firstly, we evaluate possible differences in the use of the internal and external space of the structures. Secondly, we assess the variability related to the altitudinal location of the hunting blinds. The latter has allowed discussing the use of structures to a greater extent.
\end{abstract}

Key words: Hunting blinds, technology, land use, Patagonia. 


\section{Introducción}

En los sectores mesetarios que caracterizan la Patagonia argentina, es notoria la existencia de estructuras de piedra denominadas parapetos. Los mismos pueden ser definidos como construcciones de piedra realizadas mediante la acumulación directa de rocas formando una pared, sin la utilización de argamasa. Se caracterizan por presentar, de manera general, formas circulares y semicirculares encontrándose usualmente localizadas en proximidad a cuerpos de agua y orientadas contra los vientos dominantes del oeste. En la región han sido identificadas y descriptas tanto etnohistórica como arqueológicamente (Aragone y Cassiodoro 2005, 2006; Belardi y Goñi 2006; Belardi et al. 2013, 2017; Boschin y Castillo 2005; Blanco et al. 2010; Carden et al. 2001; Cassiodoro 2011; Cassiodoro y Flores Coni 2010; Claraz 1988; Dellepiane 2014; Espinosa y Goñi 1999; Flores Coni 2014; García y Pérez de Micou 1979; Gradin 19591960; 1971; 1976; 1996; Moreno 2008; Miotti 2010; Magnin 2010; Prates et al. 2013; Vargas Gariglio et al. 2019).

Las primeras investigaciones sobre este tipo de estructuras sugieren que su funcionalidad se asociaría tanto con apostaderos de caza, como con estructuras habitacionales temporarias (Gradin 1959-1960). En función de las características de su emplazamiento vinculado a la dirección de los vientos, proximidad al agua y comportamiento de presas, algunos trabajos establecen un mayor énfasis en su asociación a la caza (Belardi et al. 2017; Cassiodoro et al. 2014; Flores Coni 2014). No obstante, considerando las particularidades del registro arqueológico asociado, no solo se los vincula con la caza y sus tareas complementarias (Cassiodoro 2011; Dellepiane y Flores Coni 2016), sino que se plantea un uso residencial temporario (Dellepiane y Cassiodoro 2019; Gradin 1971; Miotti et al. 2016). De este modo, se destaca que un análisis pormenorizado del registro directamente asociado a los parapetos es necesario para discutir con mayor sustento las características del uso de estas estructuras.

En particular, los análisis referidos a la tecnología lítica en parapetos resultan escasos. Se destacan los trabajos efectuados en el centro-oeste de la provincia de Santa Cruz que buscaron evaluar la variabilidad estratigráfica (Cassiodoro 2011), las diferencias con otros conjuntos artefactuales (Espinosa et al. 2009; Flores Coni 2018; Goñi et al. 2011-2012; Pasqualini et al. 2016) y aportar información sobre las funcionalidades de las estructuras al relacionar el registro tecnológico con el arqueofaunístico (Aragone y Cassiodoro 2006-2005; Dellepiane y Flores Coni 2016; Dellepiane y Cassiodoro 2019). Asimismo, las investigaciones de Gradin en la meseta del lago Buenos Aires (1976) son pioneras al respecto. En el mismo sentido se encuentran sus trabajos en la meseta de Somuncurá, en la provincia de Río Negro (Gradin 1971), continuadas por otros investigadores (García y Pérez de Micou 1979; Lynch y Terranova 2017; Miotti et al. 2016; Vargas Gariglio et al. 2019). 
Por otro lado, aunque en términos generales estas estructuras se encuentran agrupadas en conjuntos que van de 2 a 50 parapetos, existen escasos antecedentes tendientes a evaluar la variabilidad interna dentro de un conjunto. La primera sistematización de la variabilidad de parapetos en Patagonia fue realizada por Gradin (1959-1960) en base al registro arqueológico de la meseta del Strobel. Este autor destaca la posibilidad de hallarlas tanto de manera agrupada como aislada. Asimismo, remarca la orientación de la pared hacia el oeste, lo que brinda reparo contra los vientos y mitiga la exposición de los cazadores al olfato de las presas, así como la cercanía a cuerpos lagunares, un aspecto que ha llevado a vincularlos con el acecho e intercepción de guanacos que van a beber a ellos. Además, distingue variabilidad de formas: arco, semicírculo, en ángulo recto y circulares. En base a las diferencias en la localización y tipo de material asociado, Gradin (1959-1960) ha sugerido que el uso y la funcionalidad de estos sitios se relacionaría con estructuras habitacionales temporarias utilizadas como posibles bases de toldos o paravientos, por un lado y como apostaderos de caza, por otro. Recientemente, un análisis general de la tecnología del sitio K116 localizado en la meseta del Strobel, conformado por unas 50 estructuras, plantea variabilidad entre el conjunto de parapetos que lo conforma (Goñi et al. 2016). En este sitio habría una diferencia funcional entre las estructuras localizadas a diferentes alturas dentro del mismo. Así, en el sector de menor altura los parapetos podrían estar involucrados en instancias de acecho, encierro y caza de guanacos mientras que los localizados en un reborde más alto podrían haber sido utilizados para el procesamiento general de las presas e, inclusive, posible vivienda.

En este contexto, el objetivo principal del presente trabajo es evaluar la variabilidad interna en un conjunto de 11 estructuras de parapetos del sitio Guitarra 10, localizado en la meseta del lago Guitarra (Santa Cruz) (Figura 1). Para tal fin, en esta primera instancia, se realiza un análisis comparativo de las clases artefactuales representadas y tamaños de desechos de talla. Asimismo, se considerarán aspectos de las estructuras en sí mismas, tales como su morfología y dimensiones. La variabilidad interna de dicho conjunto de estructuras será abordada desde dos aspectos. En primer lugar, se busca evaluar si existe una tendencia al desarrollo de determinadas actividades en el espacio interno de las estructuras y otras en el espacio externo a ellas. Este punto había sido ya abordado por Gradin (1976), quien analizando materiales óseos y líticos de los parapetos de Cerro Redondo (meseta del lago Buenos Aires) plantea al espacio hacia el este de las estructuras como un sector de taller y faenamiento primario de presas. A diferencia de este autor, en este trabajo la delimitación del espacio se realiza en relación a la pared que conforma la estructura. En segundo lugar, se comparan las estructuras en función de su localización altitudinal dentro del sitio. Como fuera especificado más arriba, el sitio K116 presenta diferencias entre las estructuras localizadas a distintas alturas (Goñi et al. 2016). En el sitio Guitarra 10 las diferencias altitudinales entre parapetos son aun más marcadas que en K116, por lo cual buscamos evaluar si tal variabilidad puede ser una constante en los conjuntos de parapetos. 


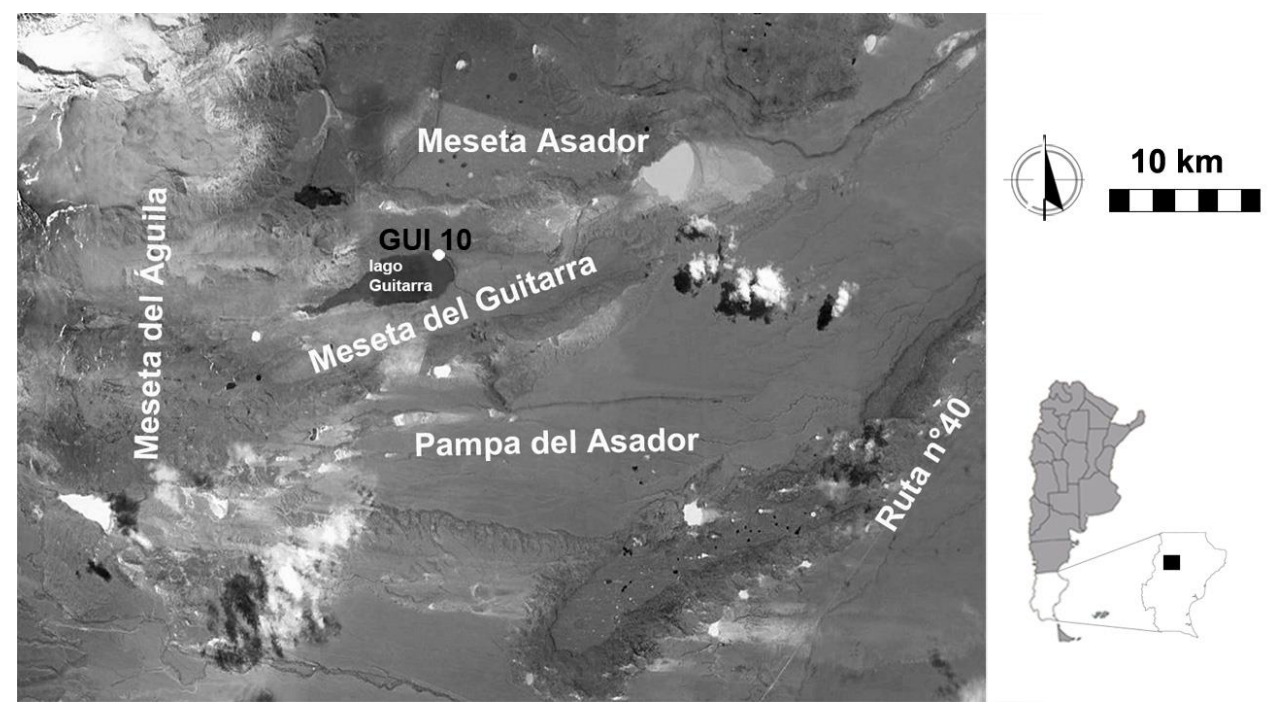

Figura 1. Ubicación del área y sitio bajo estudio.

Consideramos que el abordaje en escalas espaciales acotadas puede constituir un aporte al conocimiento sobre las particularidades de la utilización de estas estructuras tan características de algunos sectores mesetarios de Patagonia meridional.

\section{Área de estudio}

El conjunto de estructuras bajo análisis se encuentra en la meseta del lago Guitarra, ubicada en el centro-oeste de la provincia de Santa Cruz. El área constituye uno de los tantos espacios mesetarios que caracterizan a la Patagonia argentina, siendo una meseta basáltica miocénica (Ramos 2002), localizada entre los 1000 y 1100 msnm (Figura 1). Presenta un clima árido/semiárido y un ambiente de estepa herbácea que se torna inhabitable durante los meses de invierno por la gran cantidad de carga nívea.

Los relevamientos sistemáticos han permitido registrar una alta frecuencia y variedad de sitios arqueológicos en los distintos sectores de la meseta. Así, en la margen este del lago Guitarra se reconocieron dos conjuntos de parapetos, en el cañadón del río Guitarra se registraron diversas manifestaciones rupestres junto con abundante material lítico asociado a los paredones basálticos en un total de 26 sitios y, en la meseta propiamente dicha, el número de sitios es de 48 (Cassiodoro et al. 2013; Goñi et al. 2010; Re 2010; Re y Guichón 2013). Se destaca que en toda la meseta del lago Guitarra se consignaron 75 estructuras de parapetos (Cassiodoro et al. 2016). El emplazamiento de las mismas tiende a ser variable ya que se las ha registrado tanto aisladas en las planicies altas como agrupadas en el borde del cañadón y el lago Guitarra. Los fechados radiocarbónicos establecen que la meseta del lago Guitarra habría sido colonizada en el Holoceno medio y efectivamente ocupada en el Holoceno tardío (Cassiodoro et al. 2013; Goñi et al. 2010). 
Inmediatamente al sur de la meseta del lago Guitarra, a unos $7 \mathrm{~km}$, se encuentra la Pampa del Asador. Este sector se caracteriza por constituir la fuente regional de obsidiana negra y presentar una alta disponibilidad de basaltos y rocas silíceas (Espinosa y Goñi 1999). Todas estas materias primas se registran en el sitio Guitarra 10 (Cassiodoro y Flores Coni 2011). En base a la distribución de la obsidiana en la región, se sabe que el área habría sido conocida por las poblaciones desde el Holoceno temprano (Civalero y Aschero 2003). No obstante, los fechados radiocarbónicos establecen ocupaciones efectivas hacia el Holoceno tardío (Goñi et al. 2011-2012).

Considerando a los dos sectores como una misma unidad ambiental, y en función de la información arqueológica hasta el momento disponible, se ha propuesto que las actividades desarrolladas por los grupos cazadores-recolectores en Pampa del Asadorlago Guitarra, tendrían un carácter estacional, vinculadas con la caza del guanaco (Lama guanicoe) y el aprovisionamiento de materia prima lítica (Cassiodoro 2011, 2016; Goñi 2000; Goñi et al. 2011-2012; Rindel et al. 2007).

\section{El sitio arqueológico Guitarra 10}

El sitio Guitarra 10 (GUI10) se localiza en la margen este del lago homónimo a unos dos kilómetros de la naciente del río Guitarra (Figura 1). Es una concentración de 11 parapetos ubicados en dos pequeños cerros de escasa altura, a unos 40 metros de la costa actual del lago (Figura 2 A). En los distintos niveles altitudinales de uno de los cerros se localizan 10 estructuras, mientras que el parapeto restante se ubica en uno de los puntos más altos del otro cerro (Cassiodoro y Flores Coni 2010). Cabe mencionar que estos cerros son los únicos de la margen este del lago.

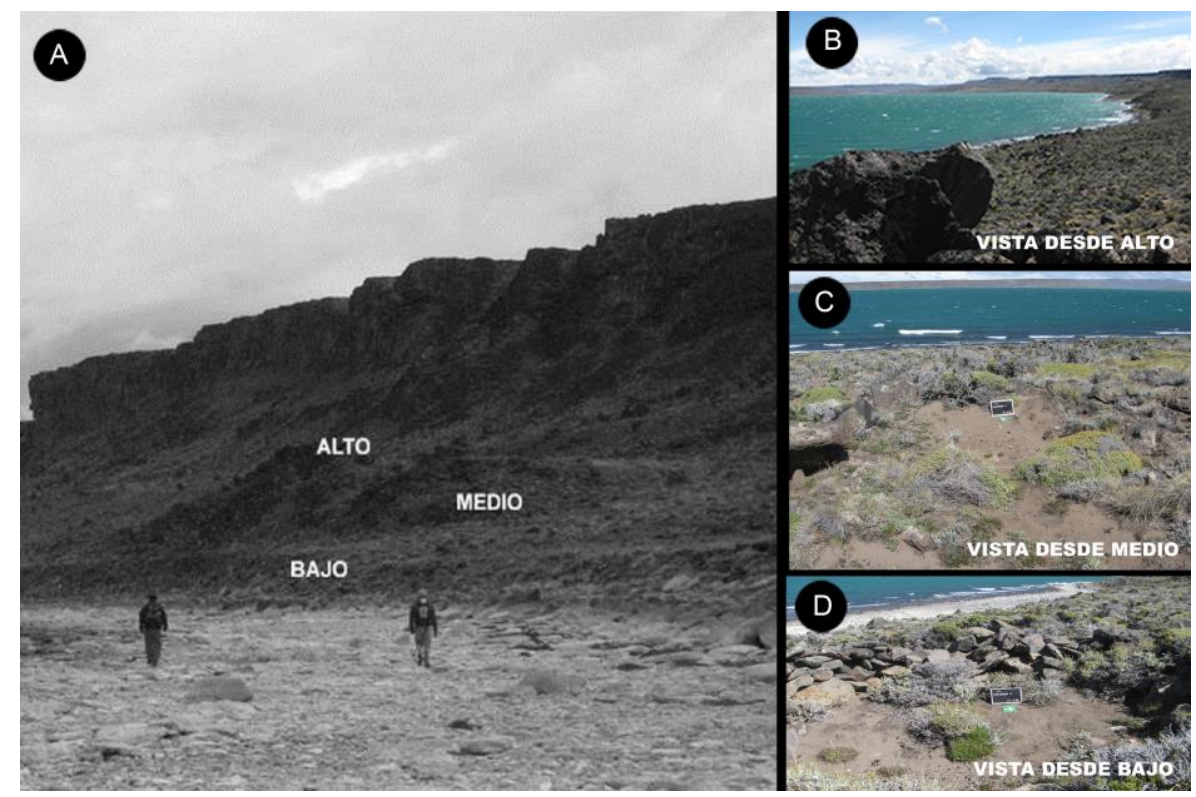

Figura 2. A- Paisaje donde se ubica el sitio GUI 10. Se indican los diferentes sectores altitudinales. B-Vista panorámica de los parapetos del sector alto del sitio. C- Vista desde parapetos del sector medio del sitio. D- Vista desde parapetos del sector más bajo del sitio. 
En función de la cercanía existente entre las estructuras y la altura en la que se encuentran, pueden establecerse distintos subgrupos (Figura 3). Por un lado, los parapetos 1 y 2 son los más cercanos al lago y están ubicados a menor altura (1133 msnm) (Figura 2 D). Un segundo subgrupo de estructuras lo constituyen los parapetos 3, 4, 5 y 6 encontrándose a una altura intermedia (1140 msnm). El modo en que están ubicados en el cerro hace que la visibilidad hacia el noroeste esté restringida (Figura 2 A y C). Los parapetos 7, 8, 9, 10 y 11 están en los sectores más elevados de los cerros (1147-1150 msnm). Cabe resaltar que la ubicación de los parapetos sobre estos sectores altos ofrece una vista panorámica general, lo que resulta en un lugar óptimo para el monitoreo de toda la costa este del lago (Figura 2 A y B y Figura 3).

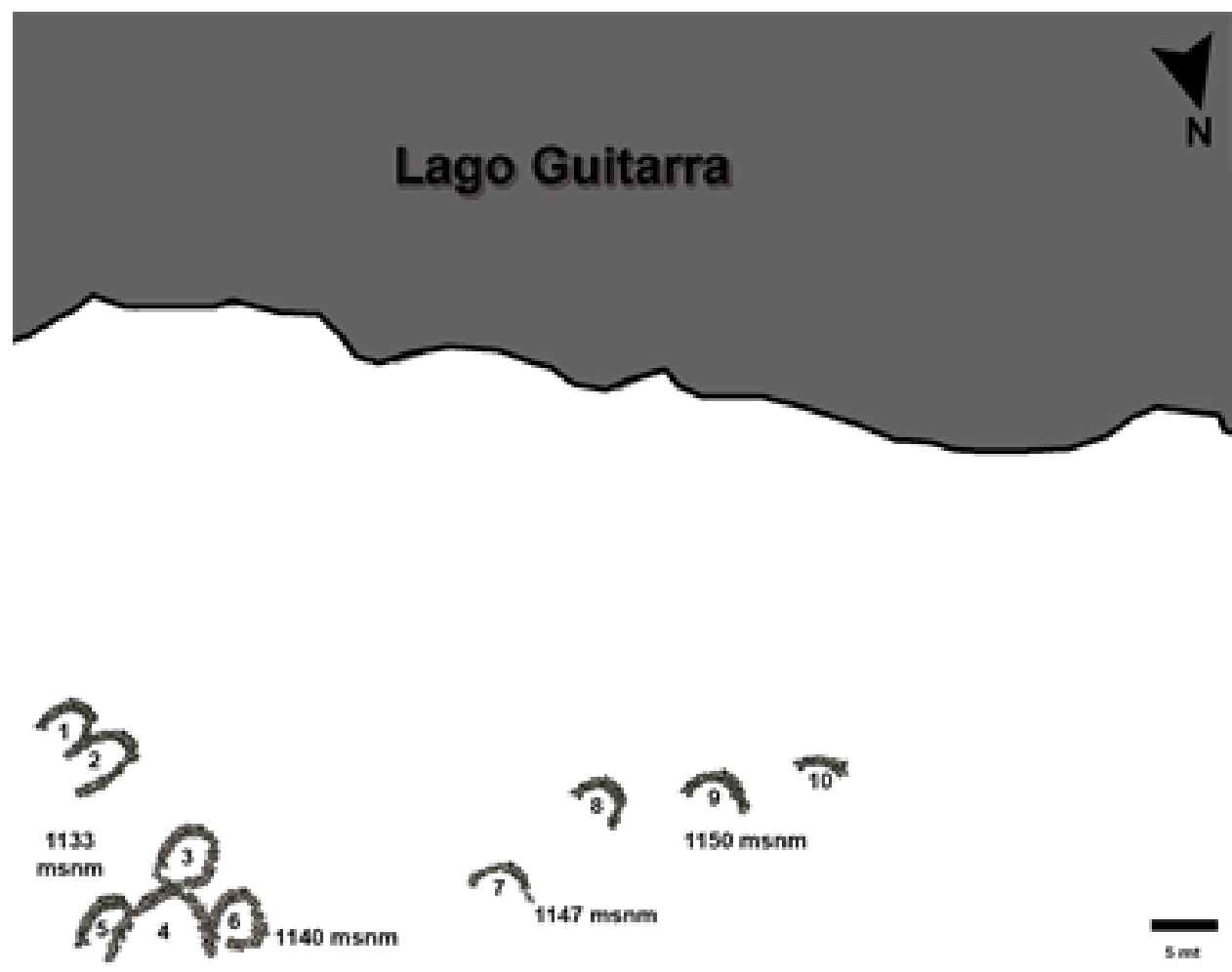

Figura 3. Ubicación de los parapetos del sitio GUI 10.

Las dimensiones internas de los parapetos son diferentes (Tabla 1), lo cual puede estar en relación con las actividades que se planifican desarrollar en los mismos. En términos generales, los parapetos que se encuentran en los sectores a mayor altura del sitio tienden a tener dimensiones menores. En cuanto a las formas de las estructuras, $45,45 \%$ de ellas son arcos y un $36,36 \%$ son semi-arcos. La morfología menos frecuente es la circular, representada únicamente por 2 parapetos (Tabla 1). Las estructuras con una forma de semi-arco están más representadas en los sectores altos del sitio. De esta manera, en términos de localización en el sitio, morfología y dimensiones, existen diferencias entre los parapetos. 
En todas las estructuras y en los espacios intermedios se ha registrado una gran cantidad de material lítico en superficie. Por su parte, el material zooarqueológico se presenta principalmente en estratigrafía y se caracteriza por la representación del guanaco con evidencias de procesamiento (termoalteración, marcas de corte y percusión) (Dellepiane 2014). El análisis específico de este material está en proceso (Dellepiane 2019). Dos estructuras han sido excavadas (Cassiodoro y Flores Coni 2010) y se cuenta con tres dataciones radiocarbónicas (Goñi et al. 2010). El nivel 2 del parapeto 2 otorgó un fechado de $507 \pm 43$ años AP (454-553 cal. AP1), el nivel 3 del parapeto 4 de $401 \pm 42$ años AP (323-497 cal. AP) y el nivel 6 de la misma estructura de $894 \pm 44$ años AP (675-818 cal. AP). Estas cronologías son coincidentes con las registradas en otros parapetos del área (Cassiodoro y Tessone 2014; Goñi 2000-2002; Goñi et al. 2011-2012; Dellepiane y Cassiodoro 2019).

\section{Materiales y métodos}

El sitio presenta una alta frecuencia de material lítico en superficie; por tal motivo, para evaluar la variabilidad del sitio se siguieron distintos procedimientos de muestreo. En primer lugar, a los fines de abordar las posibles diferencias entre el uso del espacio de cada estructura se diferenció el sector interno del externo en base a la presencia de la pared misma del parapeto. El sector externo es aquel que se encuentra inmediatamente por detrás de la pared, hacia el oeste, mientras que el interno es el que queda al reparo de la misma (Figura 4). Se realizaron recolecciones de superficie de un metro por un metro en ambos sectores. Cabe mencionar que en los parapetos ubicados en el sector más alto del sitio no existe un espacio exterior donde se pudiera efectuar tal recolección ya que todos se ubican sobre el borde de la barda del cerro. Lo mismo ocurre en el parapeto 6, localizado en el sector medio. En estos muestreos se evalúa la densidad artefactual y las clases de artefactos representadas.

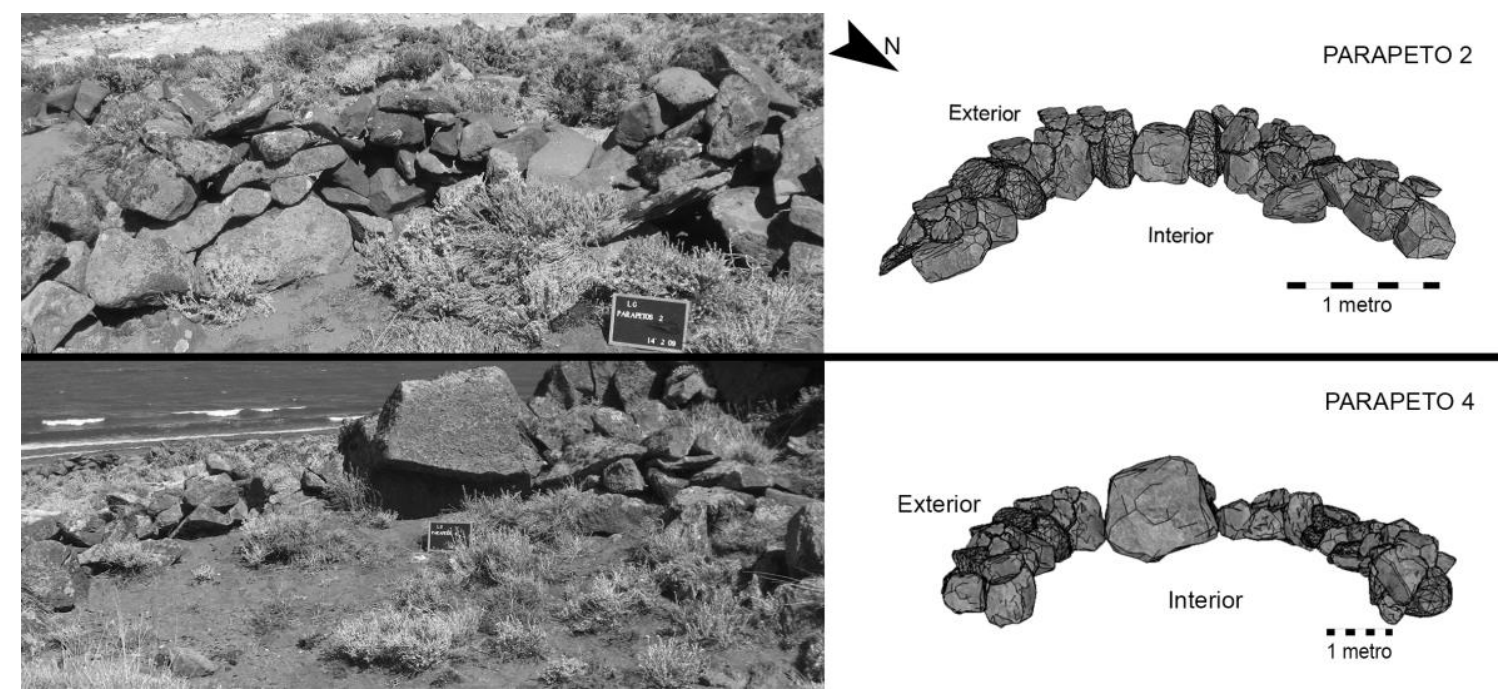

Figura 4. Foto y esquema de los parapetos 2 y 4 del sitio GUI 10. 
En segundo lugar, para analizar la variabilidad entre las estructuras, a la información anterior de los espacios internos se le agrega la proveniente de la recolección total de instrumentos y núcleos recuperados en los mismos. Teniendo en cuenta que la localización de las estructuras dentro del sitio puede estar vinculada con su funcionalidad (ver descripción ut supra), para realizar la comparación entre parapetos se los agrupó en términos de la altura a la que se encuentran en los cerros mencionados (Figuras 2 y 3 ).

La variabilidad en la tecnología asociada a parapetos también puede estar vinculada a otros aspectos como la morfología de las estructuras. No obstante, en esta instancia del análisis de GUI 10 esta perspectiva no fue abordada ya que la misma no fue observada en el estudio realizado sobre un mayor número de parapetos (n: 184) de la meseta del Strobel (Flores Coni 2018).

Los procedimientos metodológicos para el análisis de las piezas son los propuestos por Aschero (1975 rev 1983). Se especifican los grupos tipológicos de artefactos formatizados, así como las clases y tamaños de desechos de talla. A los fines de no sobredimensionar la muestra, los desechos de talla considerados para las densidades y clases son los que presentan talón. Asimismo, al momento de evaluar el tamaño de los desechos solo se tomaron en cuenta aquellos enteros.

Para evaluar la variabilidad en términos cuantitativos se estandarizan las muestras utilizando porcentajes, dado que existen diferencias en los tamaños de las mismas. Este acercamiento morfológico a los artefactos recuperados no constituye un abordaje lineal e inequívoco de la funcionalidad de los parapetos. Sin embargo, consideramos que es necesario como un primer acercamiento a la caracterización de las actividades potencialmente efectuadas en dichas estructuras. Asimismo, debe mencionarse que el accionar de procesos postdepositacionales como recolecciones hechas por pobladores actuales $^{2}$ y los procesos de erosión/ depositación de sedimentos pueden estar alterando las muestras de superficie analizadas. No obstante, la ubicación en un espacio acotado y su proximidad entre sí puede implicar que todas las estructuras hayan sido afectadas de manera similar, permitiendo la comparación.

\section{Resultados}

Uso del espacio en cada estructura: frecuencia y composición artefactual

A los fines de evaluar la variabilidad entre los parapetos del sitio y teniendo en cuenta que el espacio interior de la estructura pudo haberse utilizado de manera diferencial al espacio exterior (Binford 1978), se consideró el registro lítico recuperado en ambos espacios. Como fuera mencionado, los parapetos localizados a mayor altura dentro del sitio (estructuras 7, 8, 9, 10, 11) no tienen superficies exteriores a la pared. Lo mismo sucede en el parapeto 6. Por otro lado, en el parapeto 10 no se registró material lítico en el interior. 
Las densidades artefactuales son mayores al interior de las estructuras, principalmente en las que se encuentran en el sector bajo (Tabla 1). En contraposición, algunas densidades son menores en el sector alto. Por su parte, los parapetos 3 y 5 son los únicos con una mayor densidad artefactual en su exterior. Si se vincula las dimensiones internas de las estructuras con las correspondientes densidades artefactuales puede afirmarse que no existe una correlación entre ambas variables (r:0,05). Tampoco parece existir algún tipo de relación entre la morfología y las densidades.

\begin{tabular}{|c|c|c|c|c|c|}
\hline \multirow{3}{*}{ Localización } & GUI10 & Forma & $\begin{array}{c}\text { Superficie int. } \\
\text { del parapeto } \mathrm{m}^{2}\end{array}$ & $\begin{array}{c}\text { Densidad de } \\
\text { artefactos por } \\
\mathrm{m}^{2} \text { interior }\end{array}$ & $\begin{array}{c}\text { Densidad de } \\
\text { artefactos por } \mathrm{m}^{2} \\
\text { exterior }\end{array}$ \\
\hline \multirow{3}{*}{ Bajo } & parapeto 1 & arco & 21,20 & 172 & 116 \\
\cline { 2 - 6 } & parapeto 2 & arco & 14,52 & 199 & 36 \\
\hline \multirow{5}{*}{ Medio } & parapeto 3 & circular & 16,83 & 33 & 140 \\
\cline { 2 - 7 } & parapeto 4 & arco & 37,80 & 57 & 34 \\
\cline { 2 - 6 } & parapeto 5 & semiarco & 8,94 & 165 & 187 \\
\cline { 2 - 6 } & parapeto 6 & circular & 24,20 & 62 & 0 \\
\hline \multirow{5}{*}{ Alto } & parapeto 7 & semiarco & 25,48 & 54 & 0 \\
\cline { 2 - 6 } & parapeto 8 & arco & 6,93 & 84 & 0 \\
\cline { 2 - 6 } & parapeto 9 & semiarco & 13,51 & 0 & 0 \\
\cline { 2 - 6 } & parapeto 10 & semiarco & 4,62 & 65 & 0 \\
\cline { 2 - 6 } & parapeto 11 & arco & 11,90 & & 0 \\
\hline
\end{tabular}

Tabla 1. Características de localización, morfología y registro lítico asociado de los parapetos del sitio GUI 10 en muestreos interiores y exteriores de $1 \mathrm{~m}^{2}$.

En términos generales, en todos los conjuntos predominan ampliamente los desechos de talla y existe variedad de tipos representados (Tabla 2). En particular, las lascas angulares son las más frecuentes. Cabe mencionar la presencia de lascas de reactivación, tanto de instrumentos como de núcleos, en todos los sectores. Asimismo, en el exterior, existe una mayor representación de lascas externas (lascas primarias, secundarias y de dorso natural). También pueden destacarse las frecuencias de lascas de adelgazamiento bifacial en el interior de los parapetos 7 y 8 , localizados en el sector más alto.

Para evaluar las actividades de talla desarrolladas tanto en el interior como en el exterior, se consideraron los tamaños de los desechos enteros (Figuras 5 y 6). En todos los parapetos los tamaños más representados son muy pequeño y pequeño. La mayor representación de uno u otro de estos tamaños varía entre los espacios internos y externos de los parapetos 2, 3 y 5 . En términos generales, existe una mayor variabilidad de tamaños en el exterior de todos los parapetos, destacándose en este aspecto los parapetos 1 y 2 . 
Con respecto a los artefactos formatizados, filos naturales con rastros complementarios y núcleos, se observa que se encuentran menos representados en el exterior de los parapetos que en su interior (Tabla 3). Se destaca el parapeto 1 donde esta tendencia se invierte. Por su parte, las frecuencias de artefactos de formatización sumaria son mayores en el exterior, seguidas por los núcleos y raspadores. En el interior, los filos naturales con rastros complementarios tienen porcentajes mayores, seguidos por artefactos de formatización sumaria, núcleos y raspadores. En este sector también se encuentran puntas de proyectil y raederas.

\begin{tabular}{|c|c|c|c|c|c|c|c|c|c|c|c|c|}
\hline & \multicolumn{2}{|c|}{ Bajo } & \multicolumn{4}{|c|}{ Medio } & \multicolumn{4}{|c|}{ Alto } & \multirow{2}{*}{ Total } \\
\hline & & \begin{tabular}{|l|} 
pto 1 \\
\end{tabular} & pto 2 & pto 3 & pto 4 & pto 5 & pto 6 & pto 7 & pto 8 & pto 9 & pto 11 & \\
\hline \multirow{13}{*}{ 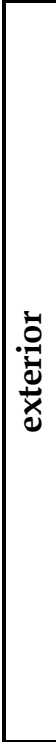 } & ls.pr & 2,04 & 10,53 & 4,00 & 4,76 & 2,04 & - & - & - & - & - & 2,90 \\
\hline & 1s.sec & - & 5,26 & 8,00 & 4,76 & 3,90 & - & - & - & - & - & 4,56 \\
\hline & ls.dn & 8,16 & 10,53 & 2,67 & 4,76 & 6,49 & - & - & - & - & - & 5,81 \\
\hline & ls.an & 55,10 & 36,84 & 58,67 & 38,10 & 49,35 & - & - & - & - & - & 51,45 \\
\hline & ls.ar & 14,29 & 10,53 & 8,00 & 19,05 & 12,99 & - & - & - & - & - & 12,45 \\
\hline & ls.pl & 4,08 & 5,26 & 5,33 & 4,76 & 3,90 & - & - & - & - & - & 4,56 \\
\hline & hoja & - & 5,26 & 1,33 & - & - & - & - & - & - & - & 0,83 \\
\hline & lámina & - & - & - & 14,29 & - & - & - & - & - & - & 1,24 \\
\hline & 1s.ind & 8,16 & - & 5,33 & - & 6,49 & - & - & - & - & - & 5,39 \\
\hline & ls. react. inst & 2,04 & - & 1,33 & 4,76 & 7,79 & - & - & - & - & - & 3,73 \\
\hline & ls.react nuc & 4,08 & 10,53 & - & 4,76 & 2,60 & - & - & - & - & - & 2,90 \\
\hline & ls. adel bif & 2,04 & - & 5,33 & - & 6,49 & - & - & - & - & - & 4,15 \\
\hline & Total exterior & \begin{tabular}{|c|}
100 \\
$(\mathrm{n}: 49)$
\end{tabular} & $\begin{array}{c}100 \\
(\mathrm{n}: 19)\end{array}$ & \begin{tabular}{|c|}
100 \\
$(\mathrm{n}: 75)$
\end{tabular} & $\begin{array}{c}100 \\
(\mathrm{n}: 21)\end{array}$ & $\begin{array}{l}100 \\
(\mathrm{n}: 77)\end{array}$ & - & - & - & - & - & $\begin{array}{c}100 \\
(\mathrm{n}: 241)\end{array}$ \\
\hline \multirow{13}{*}{ } & ls.pr & 2,30 & 0,88 & - & 2,86 & - & - & 5 & 2,78 & - & 8,70 & 1,82 \\
\hline & 1s.sec & 2,30 & 0,88 & - & 2,86 & 6,56 & 13,33 & 5 & - & - & - & 2,95 \\
\hline & ls.dn & 5,75 & 2,65 & - & 2,86 & 9,84 & - & 15 & - & 4,17 & 4,35 & 4,55 \\
\hline & ls.an & 55,17 & 46,02 & 81,82 & 40,00 & 49,18 & 46,67 & 40 & 44,44 & 37,50 & 52,17 & 48,18 \\
\hline & ls.ar & 14,94 & 21,24 & - & 25,71 & 13,11 & 6,67 & 5 & 2,78 & 12,50 & 13,04 & 14,55 \\
\hline & ls.pl & 6,90 & 12,39 & - & 5,71 & 4,92 & 16,67 & 10 & 16,67 & 20,83 & 17,39 & 10,68 \\
\hline & hoja & - & 6,19 & - & 5,71 & - & - & - & - & - & - & 2,05 \\
\hline & lámina & - & 2,65 & - & - & - & - & - & - & - & - & 0,68 \\
\hline & ls.ind & 1,15 & 3,54 & 9,09 & - & 6,56 & 3,33 & - & - & 16,67 & - & 3,41 \\
\hline & 1s. react. inst & 1,15 & 2,65 & - & 5,71 & 4,92 & 3,33 & - & - & 4,17 & 4,35 & 2,73 \\
\hline & ls.react nuc & 6,90 & 0,88 & 9,09 & 8,57 & - & 3,33 & - & - & - & - & 2,73 \\
\hline & ls. adel bif & 3,45 & - & - & - & 4,92 & 6,67 & 20 & 33,33 & 4,17 & - & 5,68 \\
\hline & Total interior & \begin{tabular}{|c|}
100 \\
$(\mathrm{n}: 87)$
\end{tabular} & $\begin{array}{c}100 \\
(\mathrm{n}: 113)\end{array}$ & \begin{tabular}{|c|}
100 \\
$(\mathrm{n}: 11)$
\end{tabular} & $\begin{array}{c}100 \\
(\mathrm{n}: 35)\end{array}$ & $\begin{array}{l}100 \\
(\mathrm{n}: 61)\end{array}$ & $\begin{array}{c}100 \\
(\mathrm{n}: 30)\end{array}$ & $\begin{array}{c}100 \\
(\mathrm{n}: 20)\end{array}$ & \begin{tabular}{|l|}
100 \\
$(n: 36)$
\end{tabular} & \begin{tabular}{|c|}
100 \\
$(\mathrm{n}: 24)$
\end{tabular} & $\begin{array}{c}100 \\
(\mathrm{n}: 23)\end{array}$ & $\begin{array}{c}100 \\
(\mathrm{n}: 440)\end{array}$ \\
\hline
\end{tabular}

Tabla 2: Desechos de talla en interior y exterior de cada estructura. Referencias: 1s.: lasca, ind.: indiferenciada, react.: reactivación, inst.: instrumentos, nuc.: núcleos, adel.: adelgazamiento, bif.: 


\begin{tabular}{|c|c|c|c|c|c|c|c|c|c|c|c|c|}
\hline & \multicolumn{2}{|c|}{ Bajo } & \multicolumn{4}{|c|}{ Medio } & \multicolumn{4}{|c|}{ Alto } & \multirow{2}{*}{ Total } \\
\hline & & pto 1 & pto 2 & pto 3 & pto 4 & pto 5 & pto 6 & pto ? & pto 8 & pto 9 & pto 11 & \\
\hline \multirow{5}{*}{ 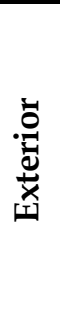 } & Afs & 33,3 & 33,3 & - & 66,6 & 100 & - & - & - & - & - & 37,5 \\
\hline & Preforma & 16,6 & - & - & - & - & - & - & - & - & - & 6,25 \\
\hline & Raspador & 16,6 & 66,6 & 33,3 & - & - & - & - & - & - & - & 25 \\
\hline & Núcleo & 33,3 & - & 66,6 & 33,3 & - & - & - & - & - & - & 31,25 \\
\hline & Total exterior & $\begin{array}{c}100 \\
(\mathrm{n}: 6)\end{array}$ & $\begin{array}{c}100 \\
(n: 3)\end{array}$ & $\begin{array}{c}100 \\
\text { (n:3) }\end{array}$ & $\begin{array}{l}100 \\
(n: 3)\end{array}$ & $\begin{array}{c}100 \\
(\mathrm{n}: 1)\end{array}$ & - & - & - & - & - & $\begin{array}{c}100 \\
(\mathrm{n}: 16)\end{array}$ \\
\hline \multirow{10}{*}{ 苞 } & Afs & - & 11,1 & 33,3 & 16,6 & 25 & - & - & - & - & 33,3 & 18,42 \\
\hline & Artef bifacial & - & 22,2 & - & - & - & - & - & - & - & - & 5,26 \\
\hline & Punta & - & 11,1 & - & - & - & 100 & - & - & - & - & 5,26 \\
\hline & Yunque/ mano & - & - & - & - & - & - & - & - & 33,3 & - & 2,63 \\
\hline & Raedera & - & 11,1 & - & 16,6 & - & - & - & - & - & - & 5,26 \\
\hline & Raspador & - & 11,1 & - & - & 41,6 & - & - & - & - & - & 15,79 \\
\hline & Lmxuso & - & - & 33,3 & - & - & - & - & - & - & - & 2,63 \\
\hline & Fncr & 100 & 33,3 & - & 33,3 & 16,6 & - & - & - & 33,3 & 66,6 & 28,94 \\
\hline & Núcleo & - & - & 33,3 & 33,3 & 16,6 & - & - & - & 33,3 & - & 15,79 \\
\hline & Total interior & $\begin{array}{c}100 \\
(\mathrm{n}: 1)\end{array}$ & $\begin{array}{c}100 \\
(n: 9)\end{array}$ & $\begin{array}{c}100 \\
(\mathrm{n}: 3)\end{array}$ & $\begin{array}{l}100 \\
(n: 6)\end{array}$ & $\begin{array}{c}100 \\
(\mathrm{n}: 12)\end{array}$ & $\begin{array}{c}100 \\
(\mathrm{n}: 1)\end{array}$ & - & - & $\begin{array}{l}100 \\
(\mathrm{n}: 3)\end{array}$ & $\begin{array}{c}100 \\
(n: 3)\end{array}$ & $\begin{array}{c}100 \\
(\mathrm{n}: 38)\end{array}$ \\
\hline
\end{tabular}

Tabla 3: Artefactos formatizados, núcleos y filos naturales con rastros complementarios en interior y exterior de cada estructura en muestreos de un metro por un metro. Referencias: afs.:

artefacto de formatización sumaria, fncr: filo natural con rastros complementarios, artef: artefacto, lmxuso: lito modificado por uso; pto.: parapeto.

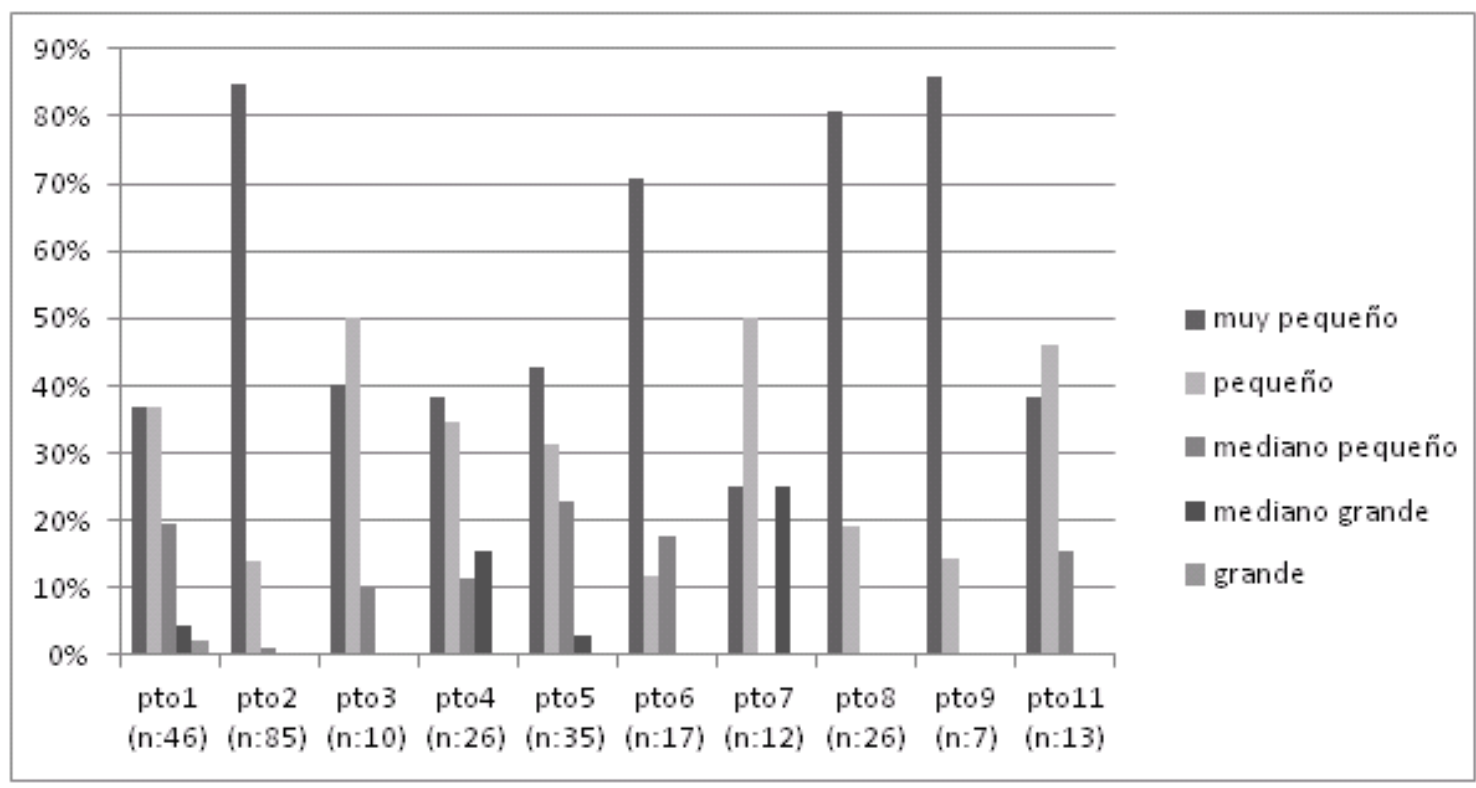

Figura 5: Tamaños desechos enteros interior. Referencias: pto.: parapeto. 


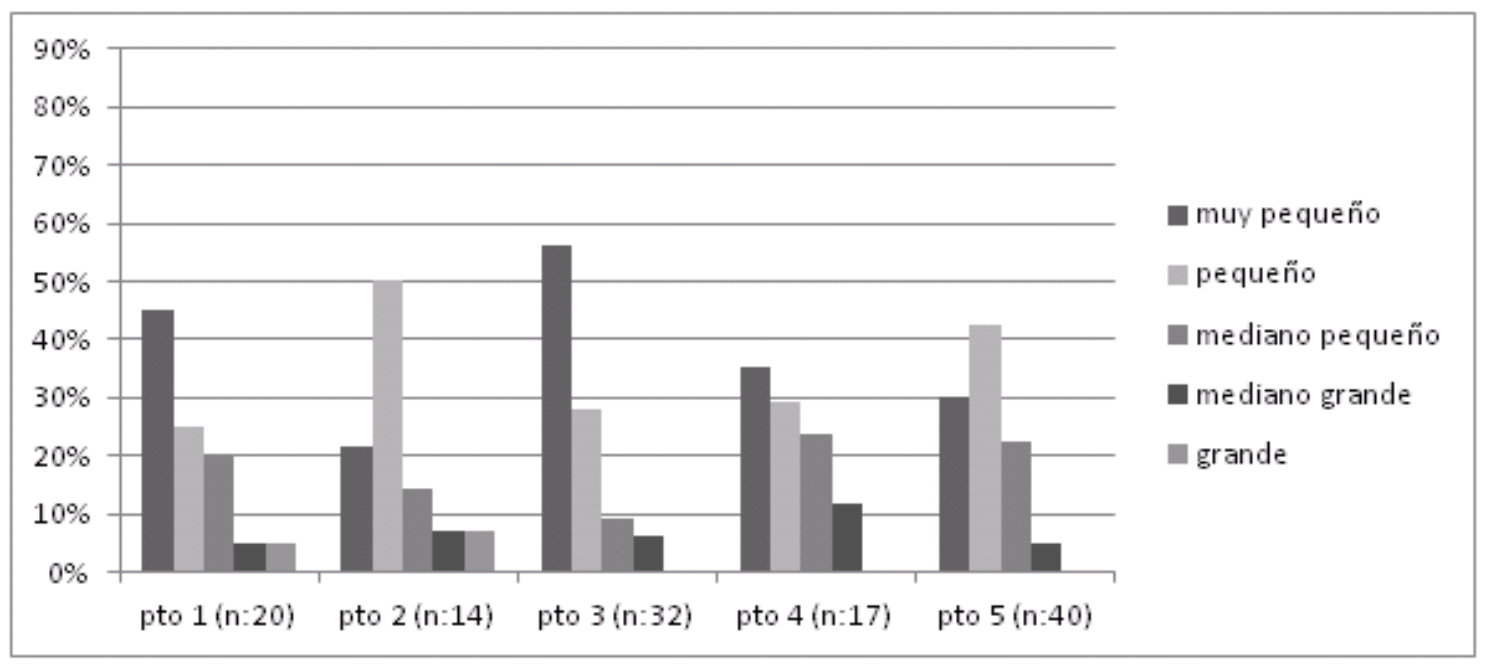

Figura 6: Tamaños desechos enteros exterior. Referencias: pto.: parapeto.

Considerando los tipos de artefactos líticos recuperados, puede plantearse la existencia del desarrollo de diferentes tareas vinculadas con la manufactura y reactivación de herramientas en ambos espacios. De este modo, en términos generales, las actividades desarrolladas tanto en el interior como en el exterior de las estructuras no serían muy diferentes. No obstante, se observa una tendencia a un mayor desarrollo de tareas vinculadas con el descortezamiento de guijarros en el exterior de los parapetos. La mayor densidad de piezas y frecuencias de artefactos formatizados al interior de las estructuras permite establecer que allí también se efectuaron actividades relacionadas con el procesamiento de recursos faunísticos.

Emplazamiento topográfico: distribución de artefactos y estructuras

Como fuera mencionado, las estructuras de GUI10 se encuentran distribuidas en diferentes alturas de dos cerros (Cassiodoro y Flores Coni 2010). En función de ello, se evalúa la variabilidad entre los parapetos considerando el total del registro lítico recuperado en su interior, es decir tanto los muestreos en un metro por un metro, como las recolecciones totales.

En primer lugar, se destacan las raederas (20,3\%), filos naturales con rastros complementarios $(18,5 \%)$ en el sector bajo; los raspadores $(23,5 \%)$ y raederas $(17,6 \%)$ en el medio y los filos naturales con rastros complementarios $(33,3 \%)$ en el alto (Tabla 4$)$. En términos generales este tool kit estaría vinculado con el desarrollo de actividades de procesamiento de recursos animales. El carácter general de las actividades habría sido el mismo en todas las estructuras. 


\begin{tabular}{|c|c|c|c|c|c|c|c|c|c|c|c|}
\hline \multirow{2}{*}{ Tipo } & \multicolumn{2}{|c|}{ Bajo } & \multicolumn{4}{|c|}{ Medio } & \multicolumn{4}{|c|}{ Alto } & \multirow[t]{2}{*}{ Total } \\
\hline & pto 1 & pto 2 & pto 3 & pto 4 & pto 5 & pto 6 & pto 7 & pto 8 & pto 9 & pto 11 & \\
\hline Afs & 20 & 10,20 & 20 & 13,16 & 15,79 & - & - & 100 & - & 33,33 & 13,45 \\
\hline Cuchillo & - & 4,08 & - & 7,89 & 5,26 & - & - & - & - & 16,67 & 5,85 \\
\hline Percutor & - & 4,08 & - & - & - & - & - & - & 20 & - & 1,75 \\
\hline Yunque/mano & - & - & - & - & - & - & - & - & 20 & - & 0,58 \\
\hline Artef. Bifacial & 20 & 4,08 & - & 2,63 & - & - & - & - & - & - & 2,92 \\
\hline Preforma bif. & - & 2,04 & - & 2,63 & - & - & - & - & - & - & 1,75 \\
\hline Punta & - & 2,04 & - & 3,95 & - & 50 & - & - & - & - & 2,92 \\
\hline Raedera & - & 22,45 & 40 & 21,05 & - & & - & - & - & - & 16,96 \\
\hline Raspador & 20 & 14,29 & - & 19,74 & 42,11 & 50 & 66,67 & - & - & 16,67 & 20,47 \\
\hline Lmxuso & - & 2,04 & 20 & - & - & - & - & - & & - & 1,17 \\
\hline Fncr & 20 & 18,37 & - & 14,47 & 15,79 & - & 33,33 & & 40 & 33,33 & 16,96 \\
\hline Núcleo & 20 & 14,29 & 20 & 14,47 & 21,05 & - & - & - & 20 & - & 14,62 \\
\hline Guijarro & - & 2,04 & - & - & - & - & - & - & - & - & 0,58 \\
\hline Total & $\begin{array}{c}100 \\
(n: 5)\end{array}$ & $\begin{array}{c}100 \\
\text { (n:49) }\end{array}$ & $\begin{array}{c}100 \\
(n: 5)\end{array}$ & $\begin{array}{c}100 \\
\text { (n:76) }\end{array}$ & $\begin{array}{c}100 \\
(n: 19)\end{array}$ & $\begin{array}{c}100 \\
(\mathrm{n}: 2)\end{array}$ & $\begin{array}{c}100 \\
\text { (n:3) }\end{array}$ & $\begin{array}{c}100 \\
(n: 1)\end{array}$ & $\begin{array}{c}100 \\
(n: 5)\end{array}$ & $\begin{array}{c}100 \\
(n: 6)\end{array}$ & $\begin{array}{c}100 \\
\text { (n:171) }\end{array}$ \\
\hline
\end{tabular}

Tabla 4: Artefactos formatizados, núcleos, filos naturales con rastros complementarios y guijarros representados en las estructuras. Referencias: afs.: artefacto de formatización sumaria; artef: artefacto; bif.: bifacial; Lmxuso: lito modificado por uso; Fncr: filo natural con rastros complementarios; pto.: parapeto.

En relación a los tamaños de los desechos de talla enteros (Figuras 5 y 6), ya se especificó que en todos los parapetos los tamaños más representados son muy pequeño y pequeño. En términos generales, en los parapetos del sector bajo y medio existe una mayor variabilidad de tamaños. Al relacionar esta información con las frecuencias de núcleos, percutores y los distintos tipos de desechos de talla, puede plantearse que todas las actividades de producción lítica se encuentran representadas en el sitio. El descortezamiento de núcleos se habría efectuado en mayor frecuencia en las estructuras localizadas en los sectores más bajos y medios.

Así, considerando en conjunto toda la información presentada, se pueden delinear algunas tendencias para cada una de las estructuras. En el parapeto 8 hay menor evidencia de descortezamiento, no se descartaron núcleos y percutores, y habría habido menor reactivación de instrumentos aunque más adelgazamiento bifacial, si bien no se observa un descarte de este tipo de piezas. Algunas de estas características las comparte con el parapeto 7. Para el parapeto 9 se observan algunas similitudes si bien se diferencia de los otros dos por el descarte de núcleos y una pieza que pudo funcionar como yunque y/o mano de moler. Algo similar ocurre con el parapeto 11, aunque en este caso habría habido más actividades de reactivación de instrumentos. El parapeto 6 se diferencia por un mayor descarte de piezas bifaciales y raspadores. Los parapetos 1, 2, 3, 4 y 5 tienen valores similares de reactivación de instrumentos, descarte de núcleos, representación de piezas con escasa formatización y raspadores. Asimismo, junto con los parapetos 6 y 7 tienen valores más altos de representación de lascas externas. 


\section{Discusión}

En Patagonia, las investigaciones sobre parapetos han tenido un mayor énfasis en los últimos años y han buscado evaluar su funcionalidad desde distintas escalas y líneas de evidencia. Así, se ha dado cuenta de diferencias entre conjuntos de parapetos (Cassiodoro 2011; Flores Coni 2018), entre algunos parapetos de un mismo conjunto (Aragone y Cassiodoro 2005; Dellepiane y Flores Coni 2016), en la estratigrafía de un mismo parapeto (Cassiodoro 2011; Vargas Gariglio et al. 2019), entre los parapetos y otros tipos de sitios (Espinosa et al. 2009; Goñi et al. 2011-2012; Pasqualini et al. 2016). En el marco de estas investigaciones, en este trabajo se buscó continuar con los abordajes tendientes a evaluar la variabilidad interna de un conjunto de parapetos (Goñi et al. 2016). En esta oportunidad se caracterizó al sitio GUI 10 constituido por un total de 11 estructuras en un espacio acotado. Las mismas tienen diferentes morfologías y dimensiones. Siguiendo abordajes previos para evaluar la variabilidad en las estructuras (Gradin 1976; Goñi et al. 2016), se consideró la ubicación altitudinal de cada una de ellas en los cerros y se evaluaron posibles diferencias entre los sectores internos y externos de cada estructura.

$\mathrm{Al}$ evaluar los sectores de cada estructura, se ha registrado que en la mayoría de los parapetos existe una mayor densidad de artefactos descartados en el espacio interior que en el exterior. La pared orientada contra los vientos del oeste ofrece reparo por lo que es esperable que al interior de las estructuras el tiempo de permanencia o tareas a realizar sea mayor (Binford 1978), y por ende también lo sea el descarte de artefactos. Al mismo tiempo, como mencionan Brooks y Yellen (1987), puede resultar esperable que las densidades artefactuales en los espacios internos de los sitios utilizados para actividades limitadas sean mayores ya que reciben una menor frecuencia de acondicionamiento de los espacios empleados. Los datos etnográficos mencionados por estos autores establecen que los parapetos están asociados a altas frecuencias de desechos artefactuales en su interior, a diferencia de lo que ocurre en bases residenciales más permanentes donde los desechos se encuentran en densidades mayores en el exterior.

Por otro lado, si bien se observan diferencias, no se ha podido establecer que los sectores internos y externos de una misma estructura hayan sido utilizados para distintas actividades. Se ha evidenciado que el desarrollo de tareas vinculadas con la producción lítica son las predominantes en todo el sitio. Como señala Binford (1978), las actividades de producción pueden superponerse a otras y encubrir la existencia de diferencias. Así, la construcción de la estructura de piedra, ya sea para establecer reparo frente a los vientos del oeste, la sujeción de toldos, o facilitar el ocultamiento de cazadores de las presas, no habría tenido demasiada injerencia en la segmentación del espacio para la producción lítica. Sin embargo, algunas discrepancias en la representación de artefactos formatizados entre ambos espacios puede llevar a pensar que otras actividades, vinculadas con el procesamiento de recursos, sí pudieron estar condicionadas por la existencia de reparo. 
En otra escala espacial, el análisis de todos los artefactos recuperados en cada parapeto buscó aportar más información a las actividades concretas llevadas a cabo en los diferentes sectores del sitio. En términos generales, la estructura artefactual de todos los parapetos no difiere demasiado entre sí. Todos comparten un tool kit compuesto por desechos de talla, núcleos, artefactos de formatización sumaria y raspadores. No obstante, existen algunas diferencias que pueden vincularse con la disposición altitudinal de cada estructura en el sitio. Así, los parapetos del sector más alto tienen una menor frecuencia de artefactos. Los artefactos formatizados descartados en ellos se corresponden con el tool kit recién mencionado. Sin embargo, la presencia de una mano/yunque, la mayor frecuencia de lascas de reducción bifacial y una menor representación de los primeros estadios de la producción lítica son los aspectos que los distinguen del resto de las estructuras. Asimismo, debe recordarse que el parapeto 10 no presentaba materiales líticos asociados y que, en términos generales, estas estructuras tienen dimensiones menores. Por su parte, los parapetos ubicados en una posición intermedia dentro del cerro y los más próximos al lago presentan mayores semejanzas entre sí. No solo tienen una mayor densidad artefactual, sino que incluyen puntas de proyectil en su estructura artefactual.

De este modo, puede proponerse que en todos los parapetos se efectuaron las mismas actividades básicas relacionadas con la manufactura y reactivación de instrumentos y el procesamiento de recursos faunísticos. Sin embargo, las características generales de las estructuras de los sectores altos (localización, dimensiones, densidad, ausencia de algunos artefactos, etc.) puede llevar a plantear que, a modo de hipótesis, ellas estarían más relacionadas con tareas de avistaje y monitoreo de presas que con la adquisición de las mismas. La actividad principal pudo haber sido la observación de las presas y la planificación de las estrategias de caza (Binford 1978). En contraposición, los parapetos de los sectores medios y bajos sí podrían vincularse de manera más directa con la obtención y procesamiento de presas. De este modo, GUI10 sigue el patrón observado en otros conjuntos de parapetos (K116) donde algunas diferencias entre estructuras artefactuales están relacionadas con la altitud a la que se localizan (Goñi et al. 2016).

En una escala de análisis mayor, no debe dejar de ser mencionado que el sitio GUI10 habría sido claramente equipado: con estructuras de piedra, artefactos poco transportables, como por ejemplo un yunque que también pudo funcionar como mano de molino, y percutores. Este equipamiento formaría parte de una estrategia de aprovisionamiento de lugares recurrentemente visitados (Binford 1979; Kuhn 1992). En el mismo sentido apuntan las cronologías disponibles que dan cuenta de la reocupación del sitio. Así, se destaca una estrategia de planificación en la utilización del sector este de la costa del lago Guitarra, donde determinado espacio habría sido utilizado de manera recurrente y configurándose como un espacio adecuado para las tareas a realizarse. En particular, los cerros en los que se emplazan los parapetos en estudio ofrecen una serie de condiciones que fomentarían su reutilización: muy buena visibilidad de todo el sector, materia prima para la construcción de estructuras y 
proximidad a una importante fuente de agua potable a nivel regional que pudo atraer la presencia de guanacos. Además, cabe recordar que este espacio está a menos de 10 km de Pampa del Asador donde existen depósitos de materia prima de excelente calidad para la talla como la obsidiana negra.

\section{Consideraciones finales}

El análisis comparativo de los artefactos líticos recuperados en parapetos ha permitido dar cuenta de potenciales actividades desarrolladas en ellos, principalmente la manufactura y reactivación de herramientas líticas y el procesamiento de recursos faunísticos. Estas actividades tienen un mayor o menor énfasis tanto en términos de los espacios que circunscriben las estructuras en sí como la localización topográfica de las mismas. De este modo, se ha hecho un aporte a la discusión sobre la variabilidad de este tipo de estructuras en un mismo conjunto.

Este sitio es sólo uno de los tantos conjuntos de parapetos que caracterizan las mesetas patagónicas (Cassiodoro et al. 2016; Flores Coni 2018). La información aquí presentada puede resultar de utilidad para delinear futuras investigaciones en otros de dichos conjuntos, afinar el análisis comparativo con sitios ya publicados, como K116 (Goñi et al. 2016), y profundizar en nuevas variables tecnológicas.

Consideramos que la información que brinda el análisis pormenorizado de estas estructuras, en términos de dimensiones, morfología y registro arqueológico asociado, resulta necesario para comprender la funcionalidad de las estructuras y su articulación en un espacio acotado. De este modo, su estudio permite ahondar en la caracterización del proceso de ocupación y poblamiento de las mesetas patagónicas, fundamentalmente durante el Holoceno tardío.

Agradecimientos: Las investigaciones fueron financiadas por los proyectos UBACYT 2014-2017 20020130100293BA y PICT-2013 n¹965. Agradecemos a la municipalidad de Gobernador Gregores y Perito Moreno, GOLDCORP, Estancias Cerro Pampa y Sierra Andía, y a quienes participaron en los trabajos de campo. Agradecemos los pertinentes comentarios de los evaluadores anónimos.

\section{Notas}

1 Edades calibradas a dos sigmas con el programa informático CALIB. REV 7.0.1 (CALIB RADIOCARBON CALIBRATION PROGRAM Stuiver y Reimer 1993), usando la curva de calibración para el Hemisferio Sur (Hogg et al. 2013).

$2 \mathrm{El}$ posible accionar de pobladores actuales en el sitio se sustenta sobre la base del registro de botellas y latas en el parapeto 6. Sin embargo, consideramos que el mismo habría sido eventual u ocasional dado que no existen caminos o rutas próximas que faciliten el acceso frecuente al sitio. 


\section{Bibliografía citada}

Aragone, A. y G. Cassiodoro

2005-2006 Los parapetos de Cerro Pampa: registro arqueofaunístico y tecnológico (noroeste de la provincia de Santa Cruz). Arqueología 13:131-154.

Aschero, C.

1975-1983 Ensayo para una clasificación morfológica de los instrumentos líticos aplicada a estudios tipológicos comparativos, Informe al CONICET. Manuscrito, Instituto Nacional de Antropología y Pensamiento Latinoamericano, Buenos Aires.

Belardi, J. y R. Goñi

2006 Representaciones rupestres y convergencia poblacional durante momentos tardíos en Santa Cruz (Patagonia argentina). El caso de la meseta del Strobel. En Tramas en la Piedra (ed. por D. Fiore y M. Podestá), pp. 85-94. WAC, SAA y AINA, Buenos Aires.

Belardi, J., S. Espinosa, G. Barrientos, F. Carballo Marina, A. Re, P. Campan, A. Súnico y F. Guichón

2013 Las mesetas de San Adolfo y Cardiel Chico: estrategias de movilidad y tácticas de caza de guanacos en el SO de Santa Cruz. En Tendencias teórico-metodológicas y casos de estudio en la arqueología de Patagonia (comp. por F. Zangrando, R. Barberena, A. Gil, G. Neme, M. Giardina, L. Luna, C. Otaola, L. Paulides, L. Salgán y A. Tívoli), pp 261-270. Museo de Historia Natural de San Rafael-SAA-INAPL, Buenos Aires.

Belardi, J.B., F. Carballo Marina, P. Madrid, G. Barrientos y P. Campan

2017 Late Holocene guanaco hunting grounds in southern Patagonia: blinds, tactics and differential landscape use. Antiquity 91 357: 718-731.

Binford, L.

1978 Dimensional analysis of behavior and site structure: learning from an Eskimo hunting stand. American Antiquity 43:330-361.

Binford, L.

1979 Organization and formation processes: looking at curated technology. Journal of Anthropological Research 35(3):255-273.

Binford, L.

1980 Willow smoke and dogs' tails: hunter- gatherer settlement systems and archaeological site formation. American Antiquity 45(1):4-20.

Blanco, R., L. Miotti y N. Carden

2010 Arte rupestre en la meseta de Somuncurá (Río Negro). Revisitando las lagunas azul y el ganso. En Arqueología Argentina en el Bicentenario de la Revolución de Mayo, XVII 
Congreso Nacional de Arqueología Argentina (ed. Por R. Bárcena y H. Chiavazza), vol. 5: 1859-1865. Zeta Editores, Mendoza.

Boschin, M. y M. del Castillo Bernal

2005 El Yamnago: del registro histórico al registro arqueológico. Revista Española de Antropología Americana 35: 9-116.

Brooks A.S y J.E. Yellen

1987 The preservation of activity areas in the archaeological record: ethnoarchaeological and archaeological work in northwest Ngamiland, Botswana. En Method and Theory for Activity Area Research. An Ethnoarchaeological Approach (ed. por S. Kent) pp. 65-106. New York.

Carden, N., M. Giardina, D. Hermo, L. Magnin y M. Vásquez

2001 Sitios de superficie, arte rupestre y estructuras de piedra en la cuenca de los zanjones Rojo y Blanco (Macizo del Deseado, Santa Cruz). En Libro de Resúmenes XIV Congreso Nacional de Argentina, p. 76, Rosario.

Cassiodoro, G.

2011 Movilidad y uso del espacio de cazadores-recolectores del Holoceno tardío: estudio de la variabilidad del registro tecnológico en distintos ambientes del noroeste de la provincia de Santa Cruz. En South American Archaeology Series 13, British Archaeological Reports (International Series), Archaeopress, Oxford.

2016 Variabilidad tecnológica en sectores altos del centro-oeste de Santa Cruz durante el Holoceno medio y tardío. Arqueología 22 (2): 335-359.

Cassiodoro, G. y J. Flores Coni

2010 Los parapetos del sitio Guitarra 10 (meseta del lago Guitarra, Santa Cruz): una aproximación tecnológica. En Arqueología Argentina en el Bicentenario de la Revolución de Mayo, XVII Congreso Nacional de Arqueología Argentina (ed. por R. Bárcena y H. Chiavazza), pp. 1871-1876. Zeta Editores, Mendoza.

Cassiodoro, G. y A. Tessone

2014 Análisis radiocarbónico y de isótopos estables en residuos cerámicos del centrooeste de Santa Cruz (Patagonia). Relaciones de la Sociedad Argentina de Antropología XXXIX (1): 293-299.

Cassiodoro, G., J. Flores Coni y J. Dellepiane

2013 Cronología y asentamiento en la Meseta del Guitarra (Santa Cruz): el sitio Cañadón Guitarra 3. En Tendencias teórico-metodológicas y casos de estudio en la arqueología de Patagonia (comp. por F. Zangrando, R. Barberena, A. Gil, G. Neme, M. Giardina, L. Luna, C. Otaola, L. Paulides, L. Salgán y A. Tívoli), pp. 297-306. Museo de Historia Natural de San Rafael-SAA-INAPL, Buenos Aires. 
Cassiodoro, G., A. Re y D. Rindel

2014 Estrategias de caza en espacios altos de Patagonia meridional: evidencia arqueofaunística, tecnológica y rupestre. En Integración de diferentes líneas de evidencia en la Arqueología Argentina (ed. por G. Cassiodoro, A. Re y D. Rindel), pp. 113-137. Aspha Ediciones, Buenos Aires.

Cassiodoro, G., R. Goñi y S. Pasqualini

2016 Variabilidad del registro arqueológico en sectores altos de Santa Cruz: tendencias generales en el uso del espacio. En Arqueología de la Patagonia de mar a mar, pp. 224-234. CIEP-Nire Negro ediciones, Coyhaique.

Civalero, T. y C. Aschero.

2003. Early Occupations at Cerro Casa de Piedra 7, Santa Cruz Province, Patagonia Argentina. En Where the South Winds Blow: Ancient Evidences for Paleo South American (ed. por L. Miotti, M. Salemme y N. Flegenheimer), pp. 141-147. Texas A\&M University Press, Texas.

Claraz, J.

1988 Diario de Viaje de Exploración al Chubut 1865-1866. Marymar, Buenos Aires.

Dellepiane, J.

2014 Zooarqueología de espacios mesetarios. Patrones de subsistencia y obtención de recursos en el centro-oeste de Santa Cruz durante el Holoceno tardío. Tesis de licenciatura. Faculta de Ciencias Sociales, Universidad Nacional del Centro de la provincia de Buenos Aires, Olavarría.

2019 Poblamiento y uso del espacio de sectores mesetarios del centro-oeste de Santa Cruz durante el Holoceno tardío. Una aproximación zooarqueológica. Tesis de doctorado. Facultad de Filosofía y Letras, Universidad de Buenos Aires.

Dellepiane J. y G. Cassiodoro

2019 ¿Estructuras de caza o campamentos temporales? Registro arqueológico del sitio Cerro Pampa 6 (Santa Cruz). En Arqueología de Patagonia: El Pasado en las Arenas (Ed. por: J. Gómez Otero, A. Svodoba y A. Banegas), pp. 361-372. Puerto Madryn, Instituto de Diversidad y Evolución Austral.

Espinosa, S. y R. Goñi

1999 Viven! Una fuente de obsidiana en la Pcia. de Santa Cruz. En Soplando en el Viento. En Actas de las III Jornadas de Arqueología de la Patagonia, pp. 177-188. INAPL-UN de Comahue, Neuquén- Buenos Aires.

Espinosa, S., R. Goñi y J. Flores Coni

2009 Aproximación tecnológica al uso de pampas y bajos en la meseta del lago Strobel. En Arqueología de la Patagonia, una mirada desde el último confin (ed. por M. Salemme, F. Santiago, M. Alvarez, E. Piana, M. Vázquez, M.E. Mansur), pp. 977- 984. Editorial Utopías, Ushuaia. 


\section{Flores Coni, J.}

2014. Análisis de la variabilidad de los parapetos en la Meseta del Strobel (Santa Cruz). Relaciones de la Sociedad Argentina de Antropología, 31: 551-557.

2018 Movilidad y uso del espacio de cazadores-recolectores en la Meseta del lago Strobel (provincia de Santa Cruz) durante el Holoceno. Una aproximación tecnológica al poblamiento de las mesetas patagónicas. Tesis de doctorado. Facultad de Filosofía y Letras, Universidad de Buenos Aires.

García, L. y C. Pérez de Micou

1979 Aproximación a un análisis funcional de parapetos pertenecientes al complejo patagoniense en la meseta de Somuncurá, provincia de Río Negro. Sapiens 4: 139-144.

Goñi, R.

2000 Arqueología de momentos históricos fuera de los centros de conquista y colonización: un análisis de caso en el sur de la Patagonia. En Desde el País de los Gigantes. Perspectivas Arqueológicas en Patagonia (ed. por J. Belardi, F. Carballo Marina y S. Espinosa), pp. 283-296. UNPA, Río Gallegos.

2000-2002 Fechados radiocarbónicos y registro arqueológico en la cuenca de los lagos Salitroso/Posadas (Santa Cruz). Cuadernos del Instituto Nacional de Antropología y Pensamiento Latinoamericano 19: 666-668.

2010 Cambio climático y poblamiento humano durante el Holoceno tardío en Patagonia meridional. Una perspectiva arqueológica. Tesis de doctorado. Facultad de Filosofía y Letras, Universidad de Buenos Aires, Buenos Aires.

Goñi, R., G. Cassiodoro, A. Re, F. Guichón, J. Flores Coni y J. Dellepiane

2010 Arqueología de la Meseta del Lago Guitarra (Santa Cruz). En Arqueología Argentina en el Bicentenario de la Revolución de Mayo, XVII Congreso Nacional de Arqueología Argentina (ed. por R. Bárcena y H. Chiavazza), pp. 1923-1928. Zeta Editores, Mendoza.

Goñi, R., G. Cassiodoro y D. Rindel

2011-2012. Poblamiento de mesetas: arqueología de Pampa del Asador y Cerro Pampa (Patagonia meridional). Cuadernos del Instituto Nacional de Antropología y Pensamiento Latinoamericano 23 (1), 21-36.

Goñi, R., G. Cassiodoro, J. Flores Coni, J. Dellepiane, A. Agnolin y R. Guichon 2016 Estrategias de caza y movilidad. Parapetos del sitio K116 (Meseta del Strobel, Santa Cruz). En Arqueología de la Patagonia de mar a mar, pp. 441-449. CIEP-Ñire Negro ediciones, Coyhaique.

Gradin, C.

1959/60 Tres informaciones referentes a la meseta del lago Strobel (prov. de Santa Cruz, Argentina). Acta Praehistorica III/IV: 144-149. 
1971 Parapetos habitacionales en la meseta de Somuncurá, provincia de Río Negro. Relaciones de la Sociedad Argentina de Antropología, V (2): 171-185.

1976 Parapetos de piedra y grabados rupestres de la meseta del lago Buenos Aires. Actas y Memorias IV Congreso Nacional de Arqueología Argentina. Revista del Museo Historia Natural de San Rafael 1:315-337.

1996 Grabados y parapetos de la zona sur de la meseta del Lago Buenos Aires (prov. de SantaCruz). En Arqueología. Sólo Patagonia (ed. por J. Gómez Otero), pp. 173-184. CENPAT- CONICET, Puerto Madryn.

Hogg, A., Q. Hua, P. Blackwell, M. Niu, C. Buck, T. Guilderson, T. Heaton, J. Palmer, P. Reimer, R. Reimer, C. Turney y S. Zimmerman

2013 Shcal13 Southern Hemisphere calibration, 0-50,000 years Cal Bp. Radiocarbon 55(4): 1889-1903.

Kuhn, S.

1992 On planning and curated technologies in the middle Paleolithic. Journal of Anthropological Research 48(3):185-214.

Lynch, V. y E. Terranova

2017 Lithic micro-wear analysis of Late Holocene tolos from Laguna Azul locality (Somuncurá plateau, Río Negro, Argentina). En 11 th International Sypmosium of Knappable Materials. From Toolstone to Stone Tools, p.202. IMHICIHU- CONICET, Buenos Aires.

Magnin, L.

2010 Distribuciones arqueológicas en la Meseta Central de Santa Cruz. Implicancias para los estudios de uso del espacio y movilidad de sociedades cazadoras-recolectoras. Tesis de doctorado. Facultad de Ciencias Naturales y Museo, Universidad Nacional de La Plata.

Miotti, L.

2010 La señal arqueológica de colonización finipleistocénica y la continuidad ocupacional en la meseta de Somuncurá, Provincia De Río Negro, Argentina. En Arqueología Argentina en el Bicentenario de la Revolución de Mayo, XVII Congreso Nacional de Arqueología Argentina (ed. por R. Bárcena y H. Chiavazza), pp. 1951-1957. Zeta Editores, Mendoza.

Miotti, L., E. Terranova, R. Blanco, L. Marchionni, D. Hermo y L. Magnin 2016 Entre basaltos y lagunas: las estructuras de piedra de la meseta de Somuncurá. Apuntes para la reflexión de los patrones de movilidad de los cazadores-recolectores. En Arqueología de la Patagonia: de mar a mar, pp. 256-266. CIEP-Ñire Negro ediciones, Coyhaique. 
Pasqualini, S., G. Cassiodoro y J. Dellepiane

2016 Logistical mobility in plateaus in Central- Western Santa Cruz, Argentina. An approach from technological, archaeofaunal and anthracological evidence. Quaternary International 422: 135-151.

Prates, L., A. Serna y E. Mange

2013 Recintos de Aguada. Estructuras de piedra prehispánicas en el este de Norpatagonia (Dpto. de Valcheta). En Actas del XVIII Congreso Nacional de Arqueología Argentina, pp. 515- 516, La Rioja.

Ramos, V.

2002 El magnetismo neógeno de la cordillera patagónica. En Geología y Recursos Naturales de Santa Cruz (ed. por M. Haller), pp. 187-199. Asociación Geológica Argentina, Buenos Aires.

$\operatorname{Re}, \mathrm{A}$.

2010 Representaciones Rupestres en Mesetas Altas de la Provincia de Santa Cruz. Circulación de Información en Espacios de Uso Estacional. Tesis de doctorado. Facultad de Filosofía y Letras, Universidad de Buenos Aires.

Re, A. y F. Guichón

2013 Las representaciones rupestres de la meseta del lago Guitarra (Provincia de Santa Cruz). En Tendencias teórico- metodológicas y casos de estudio en la arqueología de la Patagonia (ed. por A. Zangrando, R. Barberena, A. Gil, G. Neme, M. Giardina, L. Luna, C. Otaola, S. Paulides, L. Salgan y A. Tivoli), pp. 495-504. Museo de Historia Natural de San Rafael, San Rafael.

Rindel, D., G. Cassiodoro y A. Aragone

2007 La utilización de mesetas altas durante el Holoceno tardío: el sitio Cerro Pampa 2 Ojo de Agua (Santa Cruz). En Arqueología de Fuego- Patagonia. Levantando piedras, desenterrando huesos...y develando arcanos (ed. por F. Morello, M. Martinic, A. Prieto y G. Bahamonde), pp. 649-662. CEQUA, Punta Arenas.

Stuiver, M. y P.J. Reimer

1993 Extended 14C database and revised CALIB radiocarbon calibration program. Radiocarbon 35, 215-230.

Vargas Gariglio, J., E. Terranova y V. Lynch

2019 Estudio preliminar del parapeto 1 de Laguna Azul, Meseta de Somuncurá (Rio Negro, Argentina). En Arqueología de Patagonia: El Pasado en las Arenas, (Ed. por: J. Gómez Otero, A. Svodoba y A. Banegas), pp. 443-454. Puerto Madryn, Instituto de Diversidad y Evolución Austral. 